Journal of Mechanisms and Robotics. Accepted 26/03/2020

doi:10.1115/1.4046912

Copyright (c) 2020 by ASME 


\title{
Energy distribution in Dual-UAV collaborative transportation through load sharing
}

\author{
Abdullah Mohiuddin* \\ Khalifa University \\ abdullah.mohiuddin@ku.ac.ae \\ Randa Almadhoun \\ Khalifa University \\ UAE, Abu Dhabi \\ randa.almadhoun@ku.ac.ae
}

\author{
Yahya Zweiri \\ KUCARS, \\ Khalifa University of Science and Technology, \\ P.O. Box 127788, Abu Dhabi, UAE \\ Faculty of Science, \\ Engineering and Computing \\ Kingston University London \\ London SW15 3DW, UK \\ yahya.zweiri@ku.ac.ae \\ Tarek Taha \\ Algorythma's Autonomous Aerial Lab \\ UAE, Abu Dhabi \\ tarek.taha@algorythma.com
}

\begin{abstract}
In this paper, a novel dual-UAV collaborative aerial transport strategy based on energy distribution and load sharing is proposed. This paper presents the first experimental demonstration of dual-UAV collaborative aerial transport while distributing power consumption. The demonstration is performed while distributing the power consumption between two drones sharing a load based on their battery state of charge. A numerical model of the dual-hex-rotor-payload is used to validate the proposed strategy. Numerical and hardware tests were conducted to demonstrate the load distribution using multiple UAV with certain spatial configurations. Finally, collaborative aerial transport test scenarios are performed numerically and experimentally. The simulation and experimental results show the effectiveness and applicability of the proposed strategy.
\end{abstract}

\section{Introduction}

Aerial transportation of payloads via Unmanned Aerial Vehicles (UAVs) is not a new concept [1]. A commercial online shop and delivery company ${ }^{1}$ is currently evaluating the possible usage of aerial vehicles to deliver goods. A recent study showed that UAV delivery might even help in the reduction of green house emissions caused by freight industry [2]. The limitation of payload carrying capability of a single UAV can be offset by the use of multiple UAVs.

Various multi-UAV collaborative aerial transport work is present in literature providing several demonstrations of collaborative transport as discussed in [3-6]. These studies are focused on the collaboration of multiple UAVs, by either using co-ordinated motion strategies [5-7] or the leader follower approaches [8,9]. Path planning and co-operative localization while transporting a payload using multiple UAVs were presented in $[10,11]$. In $[12,13]$, haptic feedback based telemanipulation were proposed, the UAVs were equipped with $1 \mathrm{DoF}$ arm that could apply one point contact force to lift an

\footnotetext{
${ }^{*}$ Corresponding author: Mohiuddin, JMR-19-1378

${ }^{1}$ https://www.thenational.ae/business/technology/ups-drone-delivery-subsidiary-seeks-new-horizons-1.891693
} 
object. The studies mentioned above focus on the collaborative aerial manipulation without taking orientation of the payload into consideration and its effect on energy distribution between UAVs.

Several papers tackled the challenge of adjusting position and orientations of the payload in mid air [14-18], however, the uneven thrust requirements and the corresponding power distribution were not considered. In general, the orientation of the payload and the trajectory depends on the position control of the UAVs. The work presented in [19] presented a leader follower approach to achieve the desired pose without communication between UAVs by using non-zero internal force. In [7] modeling of re-configurable cable-driven parallel robots (RCDPR) was used to find the relationships between the motion of quadrotors and the motion of a payload. These studies are not focused on the effects of the payload shape on the energy distribution during collaborative transport [14-18].

Researchers have focused on collaborative transport of payloads with various shapes such as point mass, rectangular blocks and deformable linear objects [20], [21]. A point mass payload requires separation between the UAVs using longer cables as a result, the UAVs experience a pull towards each other [21]. An increase in the volume of the payload can relieve the necessity of that pulling force. However, an increase in the volume of the payload increases the implication of the center of gravity of the payload while distributing the thrust requirements. Most of the studies $[5,8,9,11]$ for collaborative transport use objects of smaller width and larger length (i.e., higher aspect ratios). In case of lower aspect ratio payload, any difference in altitude of the collaborating UAVs leads to an uneven thrust requirement, thus causing uneven energy distribution.

The thrust distribution during collaborative transport is discussed in [20,22]. The mechanism proposed by [22] regulates the thrust requirements for transporting a point mass. This regulation, however, should be performed taking the energy availability of the UAVs into consideration. The work presented in [20] discussed the need of equal load distribution while transporting deformable linear objects (DLOs), the configuration of multiple UAVs which can provide equal-load distribution was estimated using particle swarm optimization (PSO). In that case, however, it is assumed that all UAVs are identical and their battery capacities are also equivalent. The methods presented in [20,22] were not experimentally tested for verification. The studies [20,22] were also not focused on the rectangular shaped payload for thrust distribution. This study therefore focused on the rectangular shaped payload to distribute the thrust during collaborative transport to distribute the energy consumption.

Energy distribution management in multi-UAV collaborative transport is important since multi-rotors UAVs are known for low flight endurance [23] and power failure in one of the UAVs can make the transportation operation fail. This paper therefore proposes a novel experimentally verified strategy to distribute the lifting load of the jointly carried object by changing the orientation of the payload. Distribution of lifting force also enables us to regulate the power distribution between the UAVs. This distribution of power consumption can ensure mission completion when one UAV has less state of charge than the other.

The paper is structured as follows, Section 2 describes the generalized energy aware collaboration strategy for arbitrary shaped paylaod carried by $n$ UAVs. Section 2 also includes the assumptions used in this study, the multi-UAV-payload model used for the assesment and validation of the proposed strategy, the power consumption model used. Section 3 is focused on the application of energy distribution strategy for a 2D object along with the load sharing strategy, constraints and the description of dual UAV collaborative transport method. Section 4 shows the simulated results while Section 5 shows the experimental method and results.

\section{General energy aware collaboration strategy}

A general strategy for load distribution is devised for $n=2,3$ number of drones, and an arbitrary voluminous payload. It is not possible to find a closed form solution for load distribution when attitude angles of the payload are given. However, assuming the payload geometry is known, it is possible to iteratively find the required payload orientation to satisfy the load distribution requirements, subject to geometric and equilibrium constraints. The iterative strategy would require the information of the required power distribution ratio. The attitude angle of the payload around $\mathrm{x}$-axis, and y-axis, will incrementally increase. After each increment the force distribution ratio will be calculated, which will be used to calculate the power distribution ratio. If the resulting power distribution ratio will be similar to the required power distribution ratio, the iterations will stop. The process is also explained in Algorithm 1.

Let $\mathbf{a}_{\mathbf{i}}$ be the position vectors of the anchor points of the cable on the payload in the world frame $\varepsilon_{x^{\prime}, y^{\prime}, z^{\prime}}, \mathbf{r}_{\mathbf{i}}$ be the center of mass in the world frame $\varepsilon_{x^{\prime}, y^{\prime}, z^{\prime}}$, let $\tilde{\mathbf{r}_{\mathbf{i}}}$ be the position vector of center of mass in body frame $\mathcal{B}_{\tilde{x}, \tilde{y}, \tilde{z}}$, and $\tilde{\mathbf{a}_{\mathbf{i}}}$ be the anchor points in the body frame $\mathcal{B}_{\tilde{x}, \tilde{y}, \tilde{z}}$ as shown in Figure 1 . Let $\mathbf{R}$ be the rotation matrix determined by the Euler angles of the payload $\boldsymbol{\kappa}=[\alpha, \beta, \gamma]^{T}$. Given the $\mathbf{r}_{\mathbf{i}}, \tilde{\mathbf{a}_{\mathbf{i}}}$ and the attitude angles of the payload, it is possible to find $\mathbf{a}_{\mathbf{i}}$ using the relation $\mathbf{a}_{\mathbf{i}}=\mathbf{r}+\mathbf{R}\left(\tilde{\mathbf{a}_{\mathbf{i}}}-\tilde{\mathbf{r}_{\mathbf{i}}}\right)$. Starting from an arbitrary attitude angle, the world frame location of the anchor points is found. The lift forces $f_{i}$ at each anchor point in vertical axis are found using the anchor point position $\mathbf{a}_{\mathbf{i}}$, equilibrium conditions of moments around $\mathrm{x}$ and $\mathrm{y}$ axis, and equilibrium of forces in vertical axis. During hovering condition, the thrust $u_{1 i}$ can be written in the following form $f_{i}+w_{i}=u_{1 i}$ where $w_{i}$ is the weight of the drone $i$, and $f_{i}$ is the lift force applied to payload by drone $i$. This equation is simply showing that the combined thrust applied by the rotors of each UAV must be enough to support the UAV's weight and the lifting force applied to the payload by the UAV. After calculating the resulting thrust $u_{1 i}$ we can use 


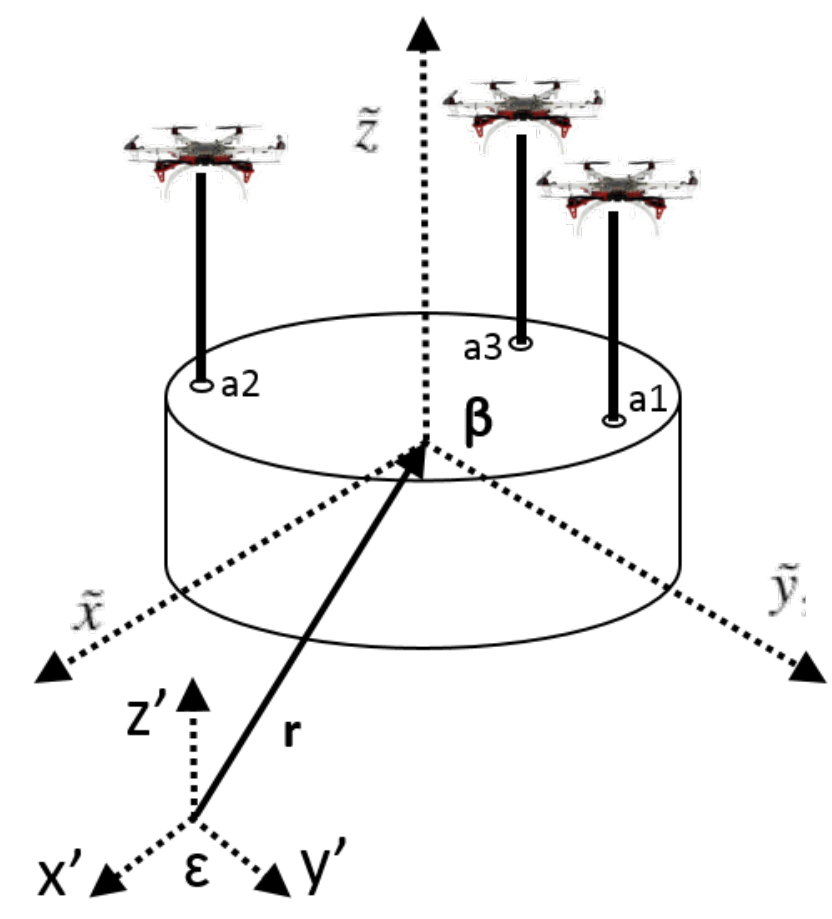

Fig. 1. Payload with respect to body and world frame along with anchor points

Eq. 7 for the calculation of the required power $p_{i}$ for each drone. The resulting power ratios, are compared with the required power ratios. The process is repeated till the payload attitude angles are found for which required power ratio is achieved. However, when $i=2$, another method can be used to distribute the load, which is explained in Algorithm 2.

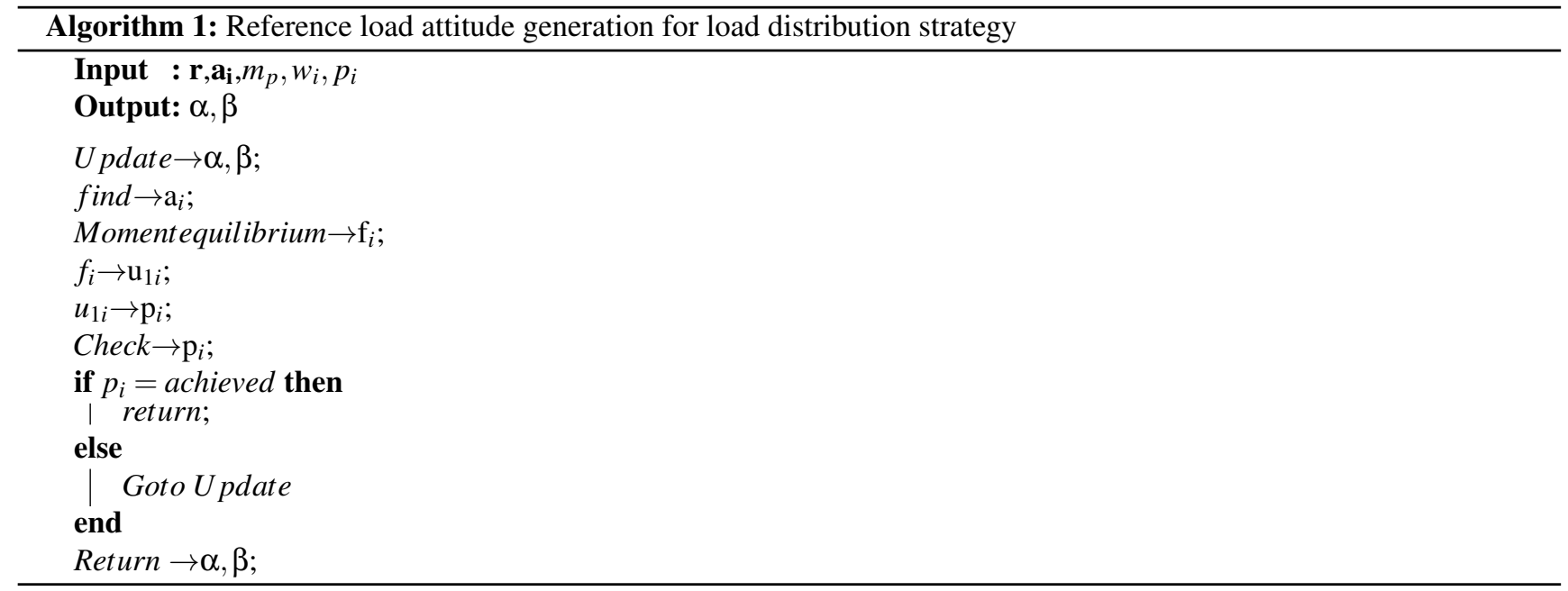

The above strategy is tested for lift load distribution, on a voluminous 3D object of mass $1 \mathrm{Kg}$ carried by $3 \mathrm{UAVs}$. The center of mass is assumed to be same as geometric center located at $[0,0,0.5]$ with respect to body frame, while the anchor points in body frame are defined as [-1,0,1],[-1 0 1],[0 1.7321 1]. Algorithm 1 was tested with several lift load ratios which it was able to process in less than $6 \mathrm{~ms}$. Lower computational costs mean that there is a possibility to incorporate the strategy for real-time implementation of the algorithm if the power consumption measurement is available in realtime. The sample runs of the code are shown in Table 1. Algorithm 1 provided not only the required attitude angles of the payload, but also the required position of the anchor points in world frame to achieve required load distribution.

\subsection{Assumptions}

Several assumptions are used for the development and testing of the strategy, which are discussed in this section.

1. We assume that the attitude dynamics of the hex-rotors is decoupled from the payload. In experiments, this is valid via 


\begin{tabular}{llcc}
\multicolumn{1}{c}{ Load ratio } & computation time $(\mathrm{ms})$ & $\alpha$ & $\beta$ \\
\hline $0.25,0.25,0.5$ & 0.03 & $28.64^{\circ}$ & $-28.64^{\circ}$ \\
$0.33,0.2,0.46$ & 0.012 & $-25.78^{\circ}$ & $-60.16^{\circ}$ \\
$0.3,0.3,0.4$ & 0.005 & $-85.94^{\circ}$ & $-11.45^{\circ}$ \\
\hline
\end{tabular}

using a small cable between the metallic strip and the supporting rod of the payload. The UAV models were simplified by assuming that the UAV structure is rigid and both Center of gravity $(\mathrm{CoG})$ and geometric center are at origin $\mathcal{U}_{\tilde{a}, \tilde{b}, \tilde{c}}$ of body frame of the UAV.

2. All aerodynamic disturbances including the lateral drag on the whole system, the thrust shielding of the payload, are ignored. A rectangular shaped payload can result in unwanted moments caused by the downwash of the air from the rotors. This aerodynamic influence of the payload to hex-rotors are ignored in simulations and was bypassed in hardware experiments via selecting an equivalent payload. The equivalent payload consists of less surface area but similar relative location of center of gravity $(\mathrm{CoG})$ from the anchor points.

3. When a point mass or low volume payload is jointly carried by two or more UAVs using cables, the UAVs experience a pull towards each other [21]. When a voluminous payload is considered with longer lengths, UAVs do not have to apply lateral forces to stay apart from each other. This also implies that the cable direction are both along the gravity direction as described in Eq. 3.

4. Propulsion system efficiency degradation during flight due to voltage supply decline is ignored. We ignore battery failsafe activation due to voltage threshold, to achieve this, experiments are performed within limits of fail-safe activation.

5. We assume equal load sharing for lateral transportation, however the lifting load is distributed amongst UAVs via our strategy. This is achieved by employing a collaborative transport strategy which works by moving the UAVs in synchronization.

\subsection{Multi-hex-rotor-payload system model}

The system consists of $n$ hex-rotors drone 01 , drone $02 \ldots$ drone-n which will be from now on referred as $i=1,2 \ldots n$, attached to a payload as shown in Fig. 1. Let $\mathbf{p}_{\mathbf{i}}=\left[x_{i}, y_{i}, z_{i}\right]^{T}$ be the position vector of the center of mass of the hex-rotor $i$ relative to the fixed inertial frame or world frame $\varepsilon_{x^{\prime}, y^{\prime}, z^{\prime}}$. The orientation of the hex-rotor $i$ is expressed in Euler angles as $\boldsymbol{\Phi}_{i}=\left[\theta_{i}, \phi_{i}, \psi_{i}\right]^{T}$ where $\theta_{i}$ is the roll angle about the $y$-axis, $\phi_{i}$ is the pitch angle about the $x$-axis, and $\psi_{i}$ is the yaw angle about the $z$-axis of the hex-rotor drone $i$. Six rotors attached to identical brush-less DC motors are rotating with a speed $\omega_{i N}$. The following equations best describe the translational and rotational dynamics model used in this paper for the hex-rotor UAVs which were modified from [24]. The model from [24] is modified to include lift forces $f_{i}$ in vertical axis. Furthermore, the payload mass was equally shared by $n \mathrm{UAVs}$ in lateral transport direction.

$$
\begin{gathered}
\left(m_{i}+n^{-1} m_{p}\right) \ddot{x}_{i}=\left(\sin \phi_{i} \sin \psi_{i}+\cos \phi_{i} \cos \psi_{i} \sin \theta_{i}\right) u_{1 i} \\
\left(m_{i}+n^{-1} m_{p}\right) \ddot{y}_{i}=\left(\cos \phi_{i} \sin \theta_{i} \sin \psi_{i}-\cos \psi_{i} \sin \phi_{i}\right) u_{1 i} \\
m_{i} \ddot{z}_{i}=\left(\cos \theta_{i} \cos \phi_{i}\right) u_{1 i}-w_{i}-f_{i} \\
I_{i x} \ddot{\phi}=\left(I_{i y}-I_{i z}\right) \dot{\theta}_{i} \dot{\psi}_{i}+u_{2 i}-J \dot{\theta}_{i} u_{5 i} \\
I_{i y} \ddot{\theta}_{i}=\left(I_{i z}-I_{i x}\right) \dot{\phi}_{i} \dot{\psi}_{i}+u_{3 i}-J \dot{\phi}_{i} u_{5 i} \\
I_{i z} \ddot{\psi}_{i}=\left(I_{i x}-I_{i y}\right) \dot{\phi}_{i} \dot{\theta}_{i}+u_{4 i}
\end{gathered}
$$

Where $i$ represents drone number. $m_{i}$ is the mass of the hex-rotor UAV and $m_{p}$ is the mass of the jointly carried payload, and $\ddot{x}_{i}, \ddot{y}_{i}, \ddot{z}_{i}$ are the translational accelerations of the hex-rotor UAV in $x^{\prime}, y^{\prime}$ and $z^{\prime}$ axes. $u_{1 i}$ is the sum of thrust $T_{i N}$ produced by all motors which is calculated as $\sum_{1}^{N=6} T_{i N}, u_{2 i}$ is the moment created by the thrust force around $x$-axis, $u_{3 i}$ is the moment created by the thrust force around $y$-axis and $u_{4 i}$ is the moment created by thrust force around $z$-axis, $u_{5 i}$ is the signed sum of speed of rotation of all propellers. The rotational inertia of the drone $i$ is expressed as $\left(I_{i x}, I_{i y}, I_{i z}\right) . J$ is the total inertia of the motor. The payload as shown in Fig. 2(a) is considered to be rigid cylinder of length $d$, with uniformly distributed mass $m_{p}$. Let $\mathbf{r}=[X, Y, Z]^{T}$ be the position vector of the center of mass of the payload relative to the fixed inertial frame $\varepsilon_{x^{\prime}, y^{\prime}, z^{\prime}}$. The orientation of the payload is expressed in Euler angles as $\mathbf{K}=[\alpha, \beta, \gamma]^{T}$ where $\alpha$ is the roll angle about the $\mathrm{x}$-axis which is described as tilt angle and is the main constituent in the load distribution strategy, $\beta$ is the pitch angle about the $y$-axis, and $\gamma$ is the yaw angle about the $z$-axis of the payload which are fixed in this case. 


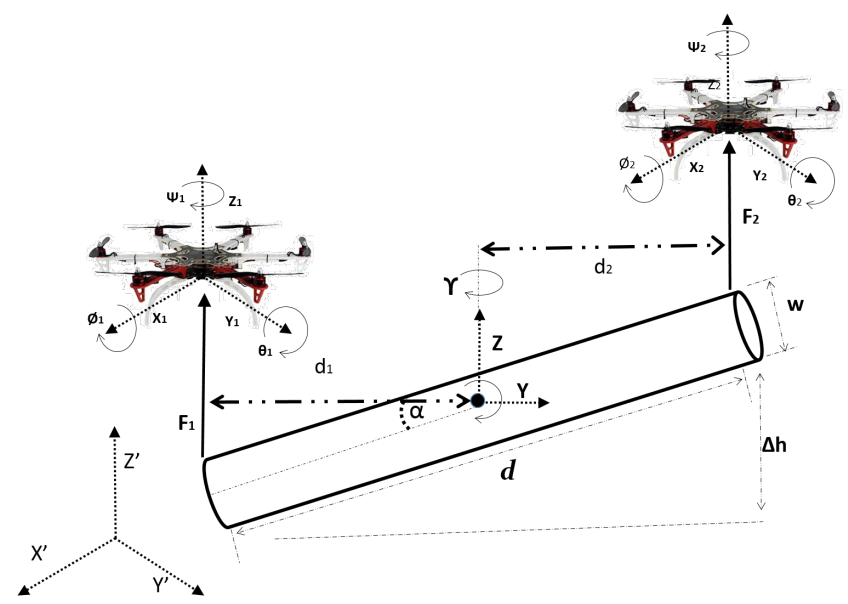

(a) Free body diagram of dual-UAV-payload system with payload in tilted orientation.

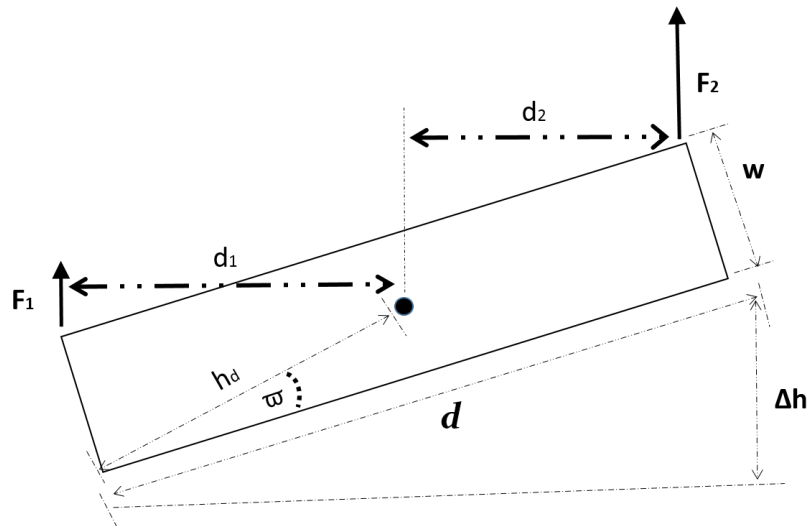

(b) Description of payload geometric parameters

Fig. 2. Payload geometric parameters and freebody diagram

\subsection{Power consumption by motors}

As in our previous work [25], an empirical equation between the rotor speed and the power consumption is obtained using curve fitting as shown in Eq. 7 , for the platform used in this study. The data-set for the empirical equation was obtained by performing lab tests using thrust stand and was also compared with [26] for the same propulsion system.

$$
P_{i N}=2 \times 10^{-8} \times \omega_{i N}^{3.3659}
$$

Where $P_{i N}$ is the power consumed by rotor $N$ of the UAV $i$, and $\omega_{i N}$ is the speed of the rotor in $\mathrm{rad} / \mathrm{s}$. The speed of the rotors is found using the relation $T_{i N}=k_{b} \omega_{i N}^{2}$ where $k_{b}=9.85 \times 10^{-6}$, where $T_{i N}$ is the thrust produced by the rotor $N$ of multirotor $i$ and $k_{b}$ is the co-efficient of lift of the propellers. The predicted power consumption of two UAVs carrying jointly equal load while hovering was compared against the experiment results as it will be shown in Section 5.

\section{Energy aware collaboration strategy for 2D object}

Given the state of charge of two UAVs to be used for joint payload transportation, the mission success and failure is tested via simulation methodology presented in this paper. Based on the state of charge of the batteries, the load can be distributed between the UAVs, using the strategy described in Algorithm 2. The steps presented in Algorithm 2 are described in this section in detail. This section will describe the system model, power distribution, load distribution, the collaborative transport strategy, and the constraints that should be considered while using this energy distribution strategy.

\subsection{Load distribution}

Assuming a payload of weight $w$ and length $d$, and considerable height is carried by two UAVs, via cable as shown in Fig. 2(a). The mass distribution is assumed to be uniform, hence center of mass of the payload is assumed to be the geometrical center of the payload. The free-body diagram of the UAV payload system is shown in Fig. 2(a). The lift force exerted on the payload by each UAV can be found by using Newton's 2nd law and summation of moments around the payload CoG. Let $f_{i}$ be the lift force exerted by the UAVs on the payload, while $d_{i}$ is the distance from the center of mass of the payload to the point of application of force $f_{i}$. Using moment summations, we know that the ratio $\frac{f_{2}}{f_{1}}$ and ratio $\frac{d_{1}}{d_{2}}$ are equal. Thus the required force distribution can be used to find the required moment arm ratios $\frac{d_{2}}{d_{1}}$. The next step is to find the UAV configuration in air that can provide the desired ratio $\frac{d_{2}}{d_{1}}$. If the tilt of the payload is $\alpha$, the sum of $d_{1}$ and $d_{2}$ can be expressed as

$$
\begin{gathered}
d_{1}+d_{2}=d \cos \alpha \\
\alpha=90-\varpi-\sin ^{-1}\left(\frac{d_{2}}{h_{d}}\right)
\end{gathered}
$$




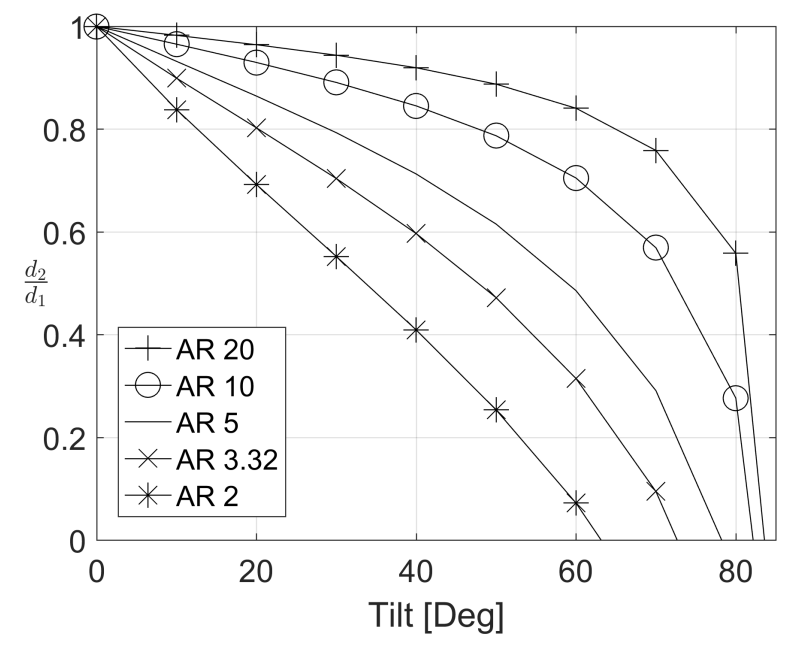

Fig. 3. Sensitivity of load-distribution potential vs tilt $(\alpha)$, for various aspect ratios (AR)

Where $h_{d}$ is the half of the diagonal length, $\varpi$ is the angle of the diagonal with the base of the payload as shown in Figure 2(b). For a given value of tilt angle $\alpha$ Eq. 8 and Eq. 9 can be solved to find the ratio, $\frac{d_{2}}{d_{1}}$, so iteratively, value of $\alpha$ can be found for which the $\frac{d_{1}}{d_{2}}$ is equal to the desired ratio. $\alpha$ can be used to find the required elevation of the drones using $\Delta h=d \sin \alpha$.

\subsection{Load distribution capacity}

The load distribution formulation was used to plot the variation of load distribution potential as represented by $\frac{d_{2}}{d_{1}}$ versus the tilt angle $\alpha$ for payloads with different aspect ratio in Fig. 3. The $y$-axis shows the values between 0 and 1 where 1 represents equal load sharing, 0.5 means that drone 02 is carrying half of the load as drone 01 , while 0 means that drone 02 is not carrying any load. Similarly $\frac{d_{1}}{d_{2}}$ can be plotted to show an increase in the load share of drone 02 while decreasing the load of drone01. Aspect ratio $(A R)$ of the payload, is the ratio of length $d$ of the payload versus the width $w_{p}$ of the payload. The load distribution sensitivity was found to vary according to the aspect ratio of the payload. As it is shown in Fig. 3, higher aspect ratios mean that the load distribution is less sensitive to the variation of $\alpha$. It is also observed that the sensitivity of load distribution increases significantly for higher aspect ratios near $90^{\circ}$ angle. At lower aspect ratios, load distribution is more sensitive to the variation of the angle $\alpha$. This implies that the potential of load distribution is higher in lower aspect ratios. Apart from that, Fig. 3 also presents the limits of load distribution, for example, a payload with aspect ratio of 2 , requires the $\alpha$ to stay below $63^{\circ}$ to have some load distribution, and after $63^{\circ}$, the load is entirely carried by one UAV. This is due to the fact that the line of action of lift force of one UAV now passes through the CoG of the payload.

\subsection{Power distribution}

Assuming that we know the required energy distribution between the two UAVs $\frac{E_{2}}{E_{1}}$, where $E_{1}$ is the energy level of drone 01 and $E_{2}$ is the energy level of drone02, we can write $\frac{P_{2}}{P_{1}}=\frac{E_{2}}{E_{1}}$ to convert the total energy consumption ratio into instantaneous power consumption ratio, where $P_{1}$ is the energy level of drone 01 and $P_{2}$ is the energy level of drone 02 . We can use Eq. 7 for the calculation of the required thrusts $u_{12}$ and $u_{11}$. During hovering condition, the thrust $u_{1 i}$ can be written in the following form $f_{i}+w_{i}=u_{1 i}$ where $w_{i}$ is the weight of the drone $i$, and $f_{i}$ is the lift force applied to payload by drone $i$. This equation is simply showing that the combined thrust applied by the rotors of each UAV must be enough to support the UAV's weight and the lifting force applied to the payload by the UAV.After obtaining the reference thrusts $u_{11}$ and $u_{12}$ via Eq. 7, we can use $f_{i}+w_{i}=u_{1 i}$ to find the required lift force ratio $\frac{f_{2}}{f_{1}}=\frac{u_{12}-w_{2}}{u_{11}-w_{1}}$. 


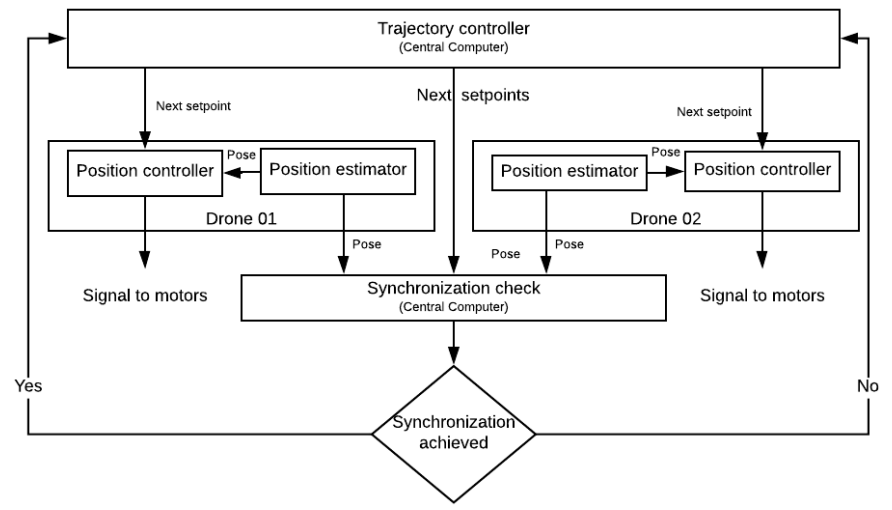

Fig. 4. Centralized co-ordinated motion collaboration strategy for multi-UAV payload transportation

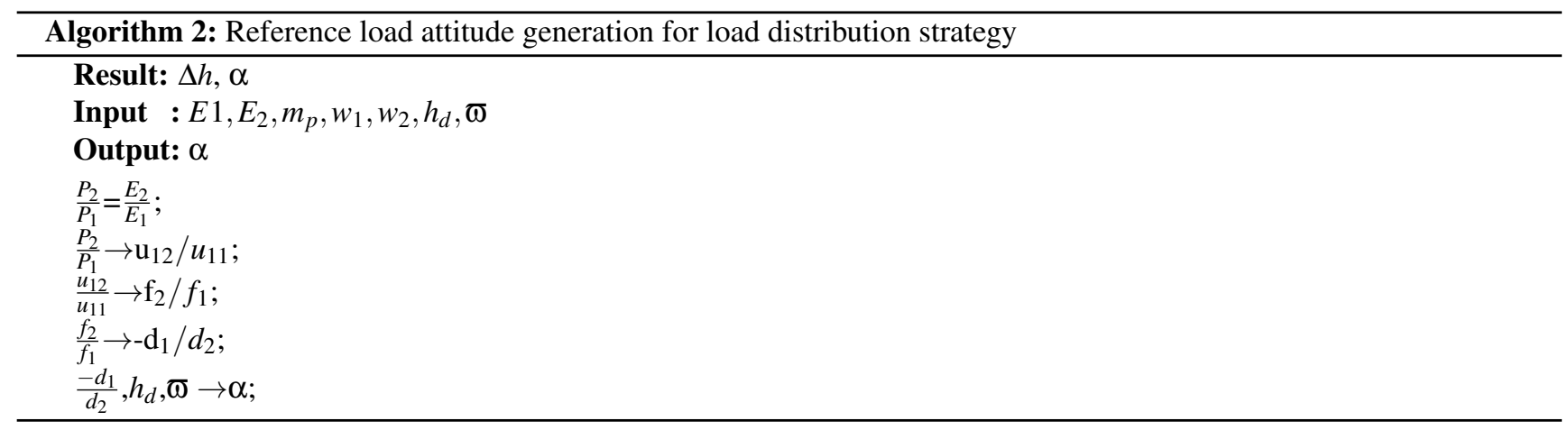

\subsection{Constraints}

Given the available energy values, we first determine the required thrust $u_{1 i}$, which should satisfy the constraints, $u_{1 i-\max }>u_{1 i}>w_{i}$, because if the required thrust is lower than the weight of the UAV itself, or higher than maximum possible thrust, the mission cannot proceed further. Another constraint is the minimum distance allowed $l_{\min }$ between two UAVs for collision prevention, and also to avoid any aerodynamic influence of one UAV to the other. $l_{\text {min }}$ can be used to find $\alpha_{\max }$ using $d \cos \alpha_{\max }>l_{\min }$. The minimum distance, can also be influenced by the possibility of any collision of the payload with the UAV rotors. The length of the gripper cable can influence the maximum tilt $\alpha_{\max }$. If $l_{s}$ be the safety factor to deal with position errors and external disturbances, $l_{g}$ is the length of the gripper, $l_{r}$ is the distance between the autopilot (Center of gravity of hex-rotor) and the rotor tip then we can use trigonometric relations to find $\alpha_{\max }$.

\subsection{Collaboration strategy}

A co-ordinated motion strategy with a centralized trajectory controller is used in this paper for testing the power distribution mechanism as shown in Fig. 4. The centralized trajectory controller is responsible for synchronized motion of the UAVs. The centralized trajectory controller, continuously monitors the error between desired pose for both UAVs and the current pose, when the error is lower than the pre-defined tolerance, the next way-points are sent to both UAVs at the same time. This collaboration strategy requires fast and reliable communication between UAVs and the central computer. The control of the multi-UAV system relies on the individual low level position controllers of each multi-rotor. It is therefore significant that these individual controllers are properly tuned. The motion controllers for both drones consist of a position controller (P), which generates velocity set-points, a velocity controller (PID), which generates the attitude set-points, and the attitude controller (PID) generates the required motor RPM set-points. This collaboration strategy was tested in software in the loop simulations (SITL) and laboratory experiments as shown in the video [27].

\section{Simulation methodology and results}

The strategy mentioned in this paper although tested on hex-rotor platform, is applicable to multi-rotors and helicopters in general. Two DJI F-550 models weighing $3.2 \mathrm{Kg}$ each and payloads were simulated using Simulink. Simulink has been used by [28,29] for modeling of a UAV. The system model as described in Sections 2.2 and 3.1 is implemented using Simulink for numerical solution of differential equations and modeling of the payload. A complete detail of the model is described in Fig. 5. The power consumption calculation used in this model is described in Section 2.3. The payload is modeled as a rigid 


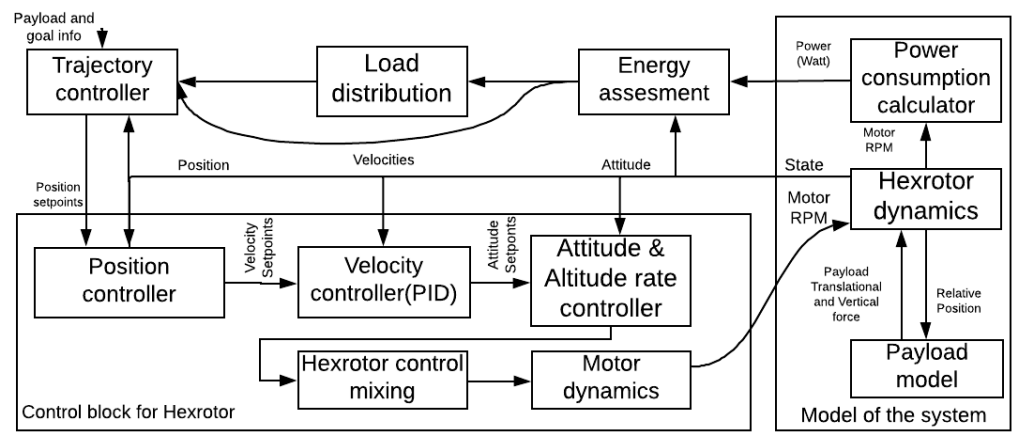

Fig. 5. A complete description of numerical simulation model components used for the verification of load distribution strategy.

body with dimensions as described in Section 3.1, which is subjected to lift force and translational force provided by the UAVs. The translational force is assumed to be equally distributed between the UAVs, however the lifting force is calculated using the altitude difference of the two drones using the formulation described in Section 3.1. The collaborative transport strategy and the motion controllers, used in this Simulink model are described in the Section 3.5. The altitude, attitude and position controllers were added to the Simulink model and tuned. All controller gains, rotor parameters, UAV inertia, UAV mass, used in the simulation were taken from [25]. Simulation tests were performed in two stages, first stage is the base case simulations, where the results were compared with the hardware experiments. The second stage is where the simulation results were extended to test extreme cases of load distribution.

\subsection{Base case simulations}

In base case simulation, the payload considered was a $586 \mathrm{~g}$ rectangular beam with dimensions $[1.6 \times 0.5 \times 0.1] \mathrm{m}$. Two DJI F-550 models named as drone01 and drone02 were considered with energy levels of $37 \mathrm{~kJ}$ and $34.5 \mathrm{~kJ}$ respectively. At first the simulation was performed with equal energy distribution. It was found via simulations that in order to transport the payload, while sharing the load equally, each drone will need $36.9 \mathrm{~kJ}$ of energy. The drone 02 with $34.5 \mathrm{~kJ}$ battery failed while transporting the payload back and forth from $-0.2 \mathrm{~m}$ to $1.4 \mathrm{~m}$ in y-axis as shown in Fig. 6(c) in red, and hence the mission was not successful.

The load distribution strategy as described in Section 3.3 was applied and a $6.2 \%$ power consumption ratio was considered. Based on this ratio, payload roll angle $\alpha=37^{\circ}$ was proposed to achieve a lift force distribution of $62 \%$ and $38 \%$. A minimum horizontal distance of $1.32 \mathrm{~m}$, with an elevation difference of $1 \mathrm{~m}$ between the drones was required to achieve this load distribution. All other constraints discussed were satisfied. When the lift force distribution was applied, the resulting power consumption by the two drones was $36.75 \mathrm{~kJ}$ and $34.25 \mathrm{~kJ}$ respectively, proving the effectiveness of the proposed strategy. The results obtained via the simulation tests are shown in first column in Fig. 6 which can be compared to the experimental results in the second column as shown in Fig. 6. The details of experimental setup and methodology is explained in Section 5. The red color represents the transportation with equal load sharing, while black color represents the collaborative transport with strategic load sharing. The dashed line represents drone 02 while solid line represents drone 01.

\subsection{Extended simulated results}

Extended simulation experiments were performed with higher load distributions and energy consumption ratios. Several collaborative transport experiments were performed with equal load sharing and unequal load sharing as shown in the Table. 2. The simulations were performed for payloads of range $0.75-1.5 \mathrm{Kg}$ mass and $[1.66 \times(0.75-1) \times 0.1] \mathrm{m}$ dimensions. Two DJI-F550 platforms with energy differences of 19.86,24.84,26,33.12\% are considered. Two cases each were simulated with and without load sharing strategy for each energy state. The Table. 2 shows a maximum of $74-26 \%$ lift force sharing and a maximum of $33.12 \%$ of energy sharing. It can be seen in Table. 2 that several configurations of energy levels result in mission failure with equal load sharing. Therefore the load sharing is performed based on the proposed strategy which leads to accomplish the mission successfully.

\section{Experimental results and discussions}

The system hardware consists of one ground station, and two DJI-F550, with Pixhawk autopilot with PX4 firmware activation and deactivation only; therefore it was powered by the same battery that powered the drone. The EPM was activated and deactivated using a ROS node. A force sensor is placed between the triangular 3D printed frame and magnetic 


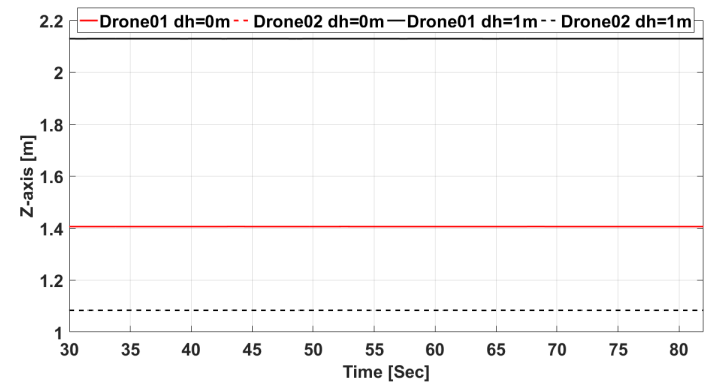

(a) Drone altitudes in simulation

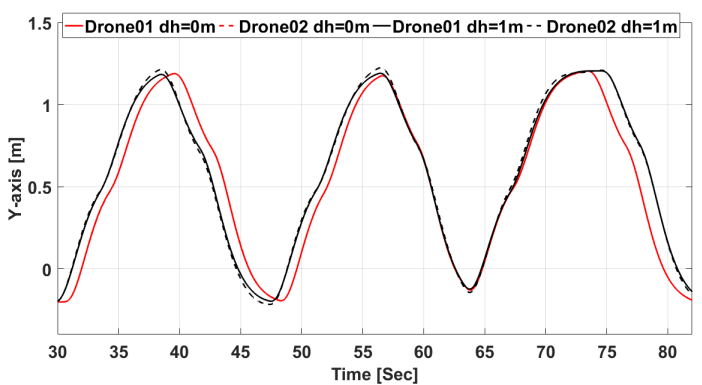

(c) Trajectory of the drones while transporting the payload in simulations

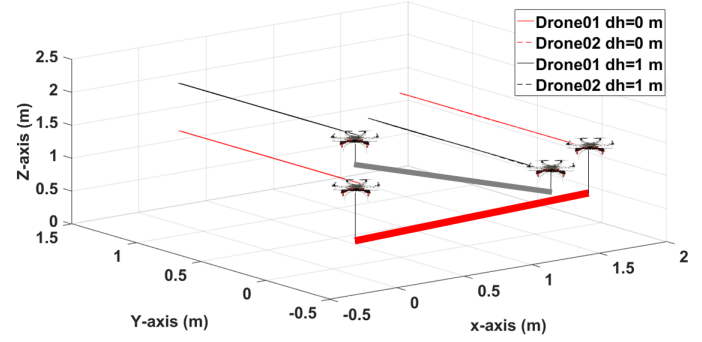

(e) Relative positions of the drone during simulations

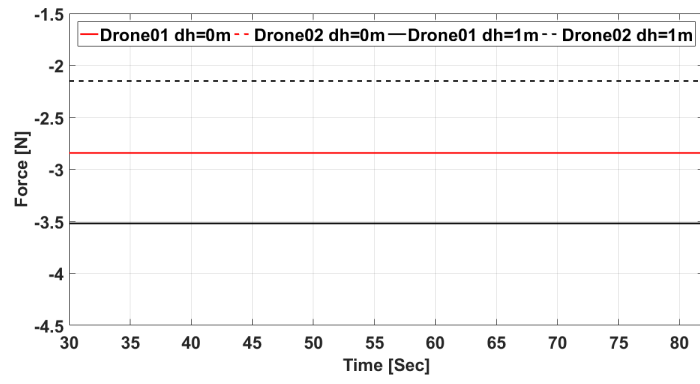

(g) Lift force distributions in simulation

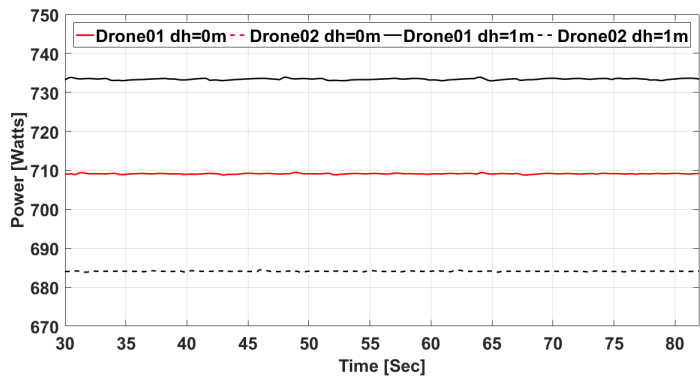

(i) Power consumptions in simulation

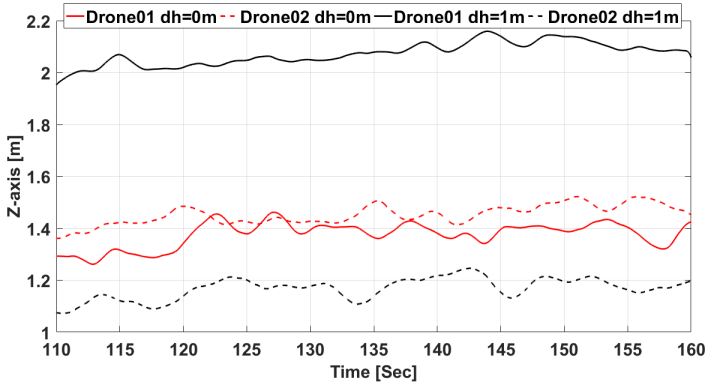

(b) Drone altitudes in experiment

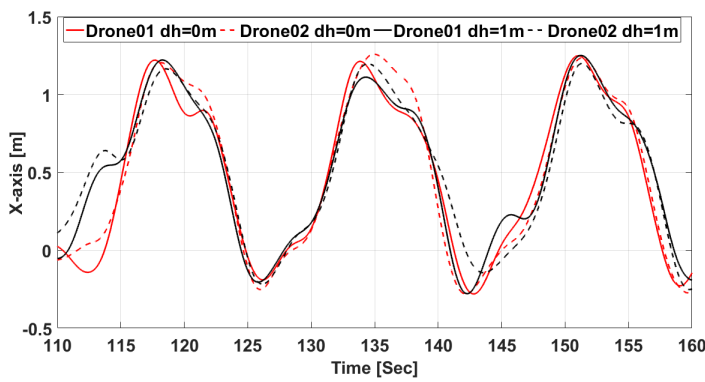

(d) Trajectory of the drones while transporting the payload during experiments

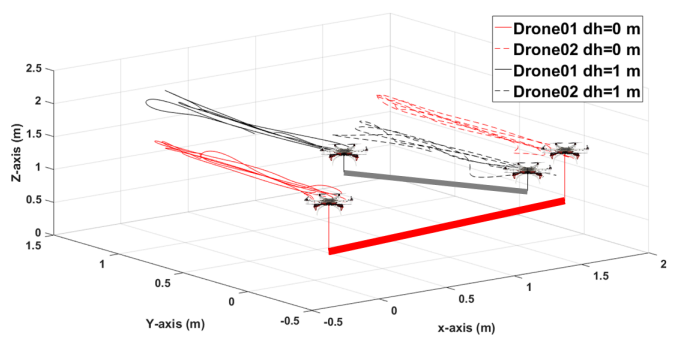

(f) Relative positions of the drone during experiments

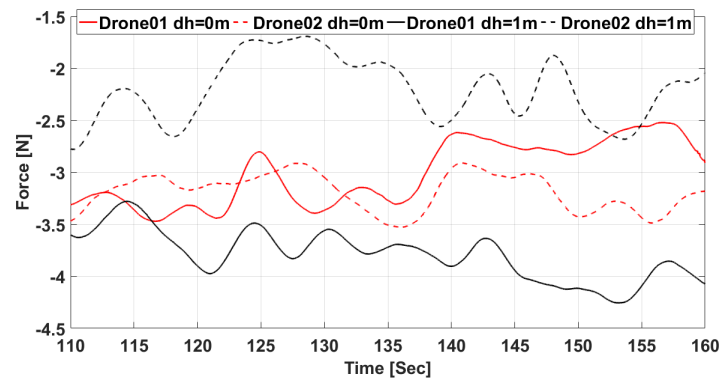

(h) Lift force distributions in experiment

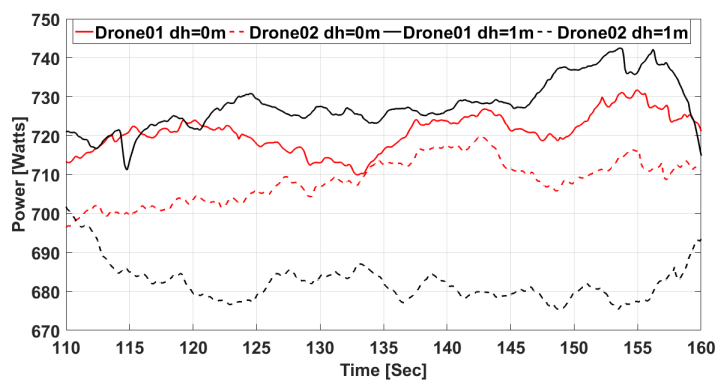

(j) Power consumptions in experiment

Fig. 6. First and second column represent similar experimental and simulation scenarios 


\begin{tabular}{llllcl} 
Payload mass & \multicolumn{1}{c}{ shape } & \multicolumn{1}{c}{$\Delta E$} & \multicolumn{1}{c}{$\alpha$} & \multicolumn{1}{c}{$\mathbf{f}_{\mathbf{1}} / \mathbf{f}_{\mathbf{2}}$} & outcome \\
\hline $1 \mathrm{Kg}$ & {$[1.66 \mathrm{~m} \times 1 \mathrm{~m} \times 0.1 \mathrm{~m}]$} & $0 \%$ & $0^{\circ}$ & $50 \%-50 \%$ & Failure \\
$1 \mathrm{Kg}$ & {$[1.66 \mathrm{~m} \times 1 \mathrm{~m} \times 0.1 \mathrm{~m}]$} & $24.84 \%$ & $46^{\circ}$ & $81 \%-19 \%$ & Success \\
$1.5 \mathrm{Kg}$ & {$[1.66 \mathrm{~m} \times 1 \mathrm{~m} \times 0.1 \mathrm{~m}]$} & $0 \%$ & $0^{\circ}$ & $50 \%-50 \%$ & Failure \\
$1.5 \mathrm{Kg}$ & {$[1.66 \mathrm{~m} \times 1 \mathrm{~m} \times 0.1 \mathrm{~m}]$} & $33.12 \%$ & $46.29^{\circ}$ & $81 \%-19 \%$ & Success \\
$1.5 \mathrm{Kg}$ & {$[1.66 \mathrm{~m} \times 0.75 \mathrm{~m} \times 0.1 \mathrm{~m}]$} & $0 \%$ & $0^{\circ}$ & $50 \%-50 \%$ & Failure \\
$1.5 \mathrm{Kg}$ & {$[1.66 \mathrm{~m} \times 0.75 m \times 0.1 \mathrm{~m}]$} & $26 \%$ & $46.29^{\circ}$ & $74 \%-26 \%$ & Success \\
$0.75 \mathrm{Kg}$ & {$[1.66 \mathrm{~m} \times 1 \mathrm{~m} \times 0.1 \mathrm{~m}]$} & $0 \%$ & $0^{\circ}$ & $50 \%-50 \%$ & Failure \\
$0.75 \mathrm{Kg}$ & {$[1.66 \mathrm{~m} \times 1 \mathrm{~m} \times 0.1 \mathrm{~m}]$} & $19.86 \%$ & $46.29^{\circ}$ & $81 \%-19 \%$ & Success \\
\hline \hline
\end{tabular}

gripper as shown in Fig. 7(b). The force sensor (FSE1001) is uniaxial force sensor which transmits the force values using the USB port of the computer mounted on the drone. The data received by the force sensor is converted into force values using a ROS node and is published on a ROS topic with a frequency of $150 \mathrm{~Hz}$. A ground station with ROS Master works as centralized station to communicate and command both drones via wireless connection. Three sets of experiments were conducted to validate the load sharing method for collaborative transport. First, baseline tests were performed to benchmark the power consumption of both drones as explained in subsection 5.1. After that, the payload was lifted and kept in hover by the drones while sharing the load in the second set of experiments which are explained in subsection 5.2. In the last experiment, collaborative transport was performed with equal and uneven load sharing as described in subsection 5.3.

\subsection{Baseline test}

Firstly a baseline hovering power consumption test was performed. In this test drone 01 and drone 02 took off together autonomously and reached the same altitude. The drones were hovering without any external payload while power consumption was recorded which illustrated that the power consumption profile is similar for both drones as shown in Fig. 8. A similar test was conducted where both drones were jointly carrying the payload while sharing the payload equally. It can be observed in the Fig. 8 that both drones are now consuming an increased amount of power due to payload addition, and the amount of power consumed by both drones is similar.

\subsection{Load sharing in hover}

The aim of load sharing experiment is to observe the effect of spatial configuration of the drones on the power consumption. A payload equivalent of $[1.6 \times 0.5] \mathrm{m}$ of mass $580 \mathrm{~g}$ is constructed using two lightweight aluminum rods of length $0.25 \mathrm{~m}$ rigidly fixed perpendicular to a $1.6 \mathrm{~m}$ rod of rectangular cross-section as shown in Fig. 7(d). The aspect ratio of the equivalent selected payload is 3.32 . The mass of the $1.6 \mathrm{~m}$ rod being significantly greater than the $0.25 \mathrm{~m}$ aluminum rods makes it possible to have the center of mass of the payload at the center of the $1.6 \mathrm{~m}$ rod, mimicking a beam of $[1.6 \times 0.5] \mathrm{m}$ of mass $580 \mathrm{~g}$. The payload is already attached to the gripper of the drones. The experiment consists of autonomous takeoff of the drones with payload where both drones are at the same altitude, and then achieving desired spatial configuration for both drones. The altitude difference of the two drones was selected with an increment of $0.25 \mathrm{~m}$ from 0 to $1.25 \mathrm{~m}$. The experiments were constrained to avoid ground effects while staying within the range of Optitrack system and the payload weight chosen based on the payload limitations of the drones. Further increase in altitude difference from $1.25 \mathrm{~m}$ resulted in violation of geometric constraint as shown in the experiment video ${ }^{2}$. The drones start by taking-off together with the payload, then achieving same altitude, and wait for 5 seconds to stabilize in the same altitude. After that the desired altitude difference is achieved and maintained for 150 seconds. The instantaneous power consumption for all cases for both drones is shown in Fig. 9(b), which shows the incremental difference in power consumption by both drones with incremental change in the altitude. Total energy consumption is then calculated for each drone. A comparison is made between the percentage difference of total energy consumption between the two drones for each tilt angle $\alpha$ as shown in Fig. 9(a). The total energy for each case is found by integrating the instantaneous power over the time of flight. After finding the total energy for each

${ }^{2}$ https://youtu.be/o2K4BWfRGHk 


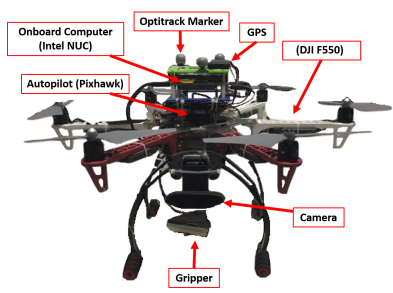

(a) The drone DJI F550 experimental platform

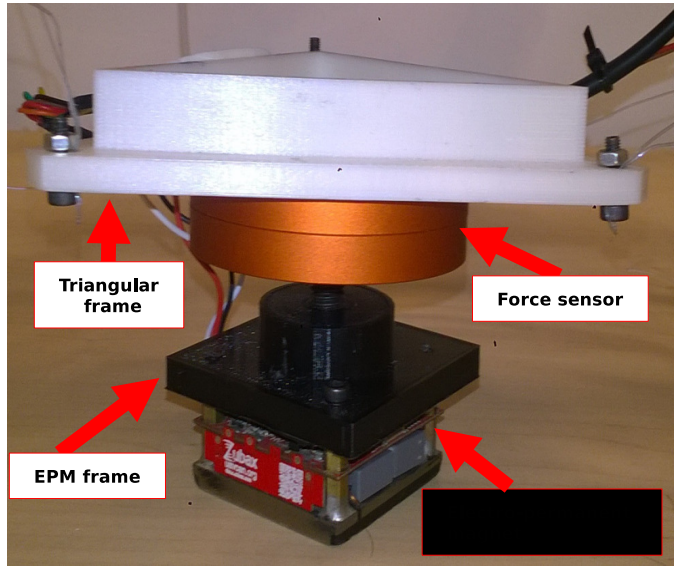

(b) EPM gripper with force sensor

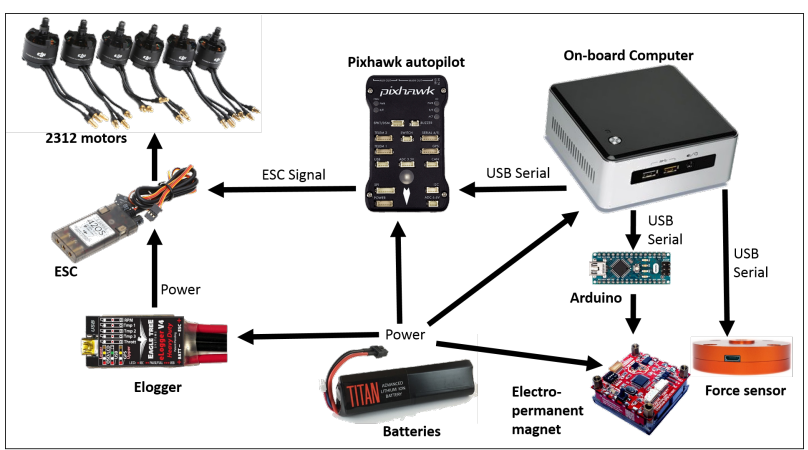

(c) The connections between the computer, autopilot, EPM force sensor and eloggers

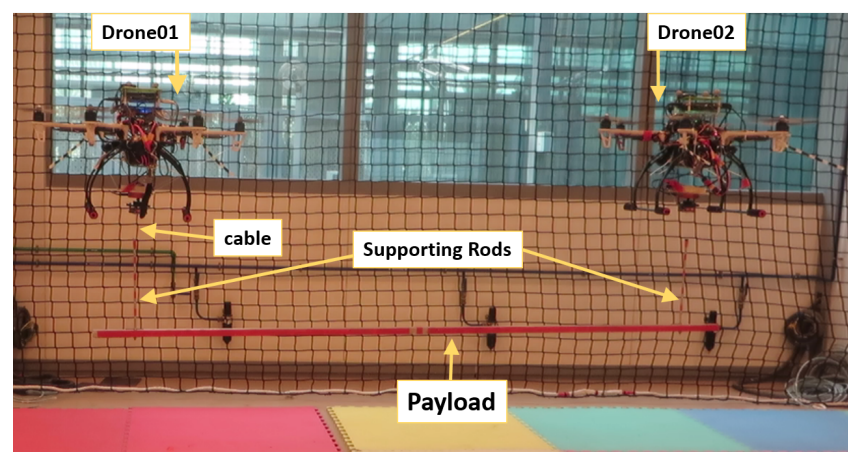

(d) Drone01 and drone02 during experiment

Fig. 7. A description of the hardware used in the experiments. The payload, hex-rotors, gripper with force sensor and EPM is also shown

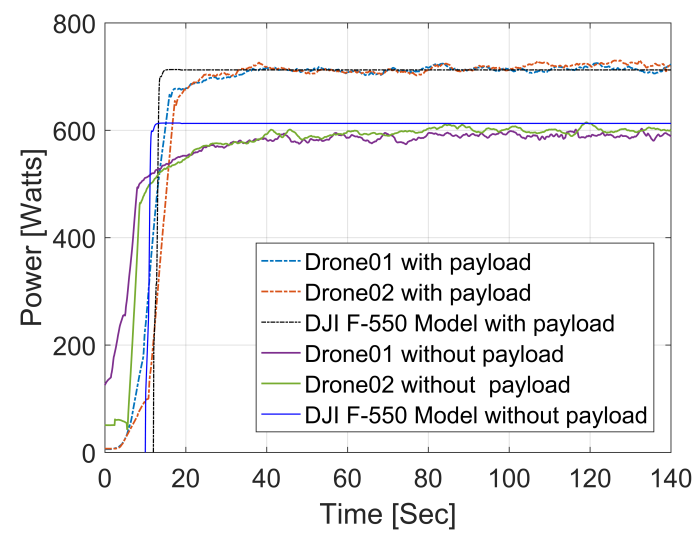

Fig. 8. Instantaneous power and energy consumption by both drones, with and without payload, where dashed lines represent energy consumption of drones with payload

drone, the percentage energy consumption difference is calculated and plotted in Fig. 9(a). It can be seen in Fig. 9(a) that the experimental results are in agreement with the simulated results. These experiments provide the proof of concept of the load distribution which can be achieved by manipulating the orientation of the payload. It is also observed here that power consumption varies with time, showing an increase in power consumption of drone carrying heavier load. Future work will include improvement of the strategy to account for such increase. A video demonstration of the whole set of experiments can be accessed via link ${ }^{2}$. 


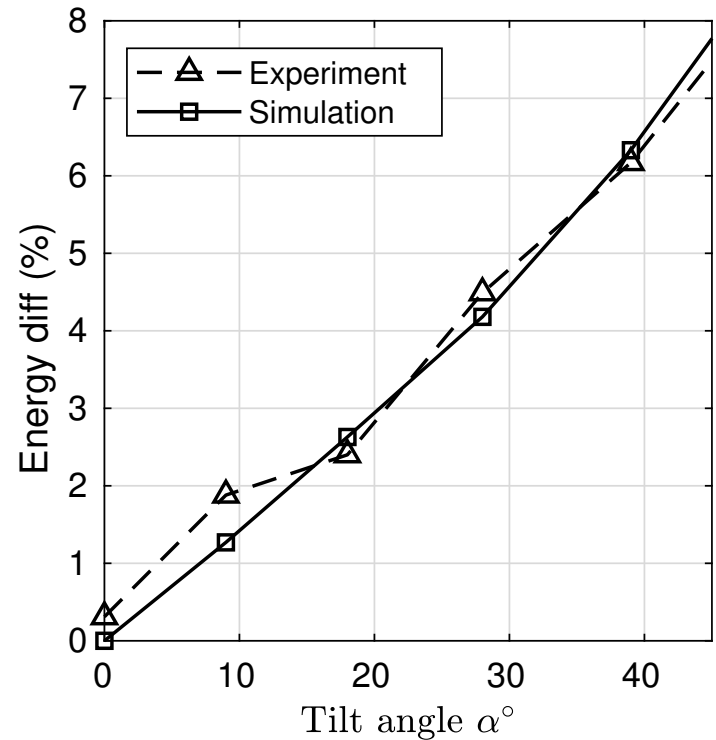

(a) $\Delta E$ vs tilt angle $\alpha$ between two drones for simulation and experiments

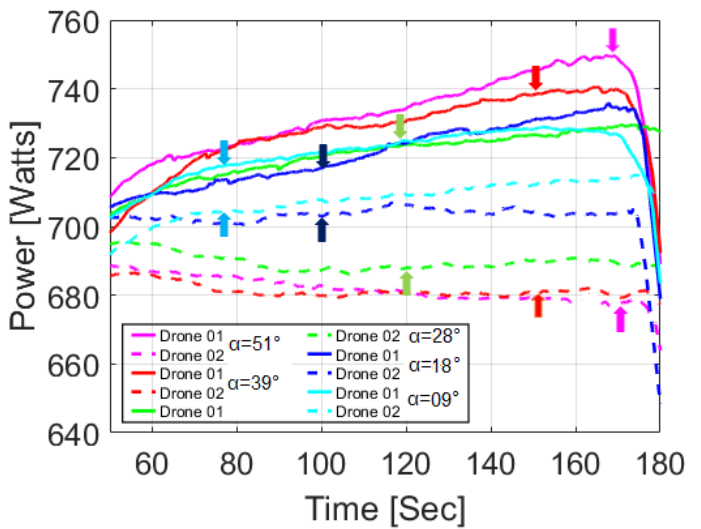

(b) Power consumption at various tilt angles for corresponding altitude differences of $0.25-1.25 \mathrm{~m}$, arrows are used to hint the corresponding pair of drones during one test

Fig. 9. Power consumption during load sharing in hover

\subsection{Load sharing in collaborative transport}

Two tests were conducted to demonstrate the collaborative transport via load sharing. In these test, drones autonomously takeoff while jointly carrying the payload to the transportation altitude. In the first test, the collaborative transport was performed via equal load sharing i.e. the transportation altitude set-point was the same for both drones. In the second test, collaborative transport was performed via uneven load distribution i.e drone 01 was given a high altitude set-point compared to drone 02 . The second test was aimed to distribute the lifting load $62 \%$ and $38 \%$ to drone 01 and drone 02 respectively, to achieve a $6.2 \%$ distribution of energy consumption. This means that if the energy level of drone02 is $6.2 \%$ lower than drone01, the distribution of load will still make the transportation possible. This test is the replication of the simulation test performed in the previous Section 4.1 with the models using the same hardware. Both tests successfully demonstrated the to and fro transportation of a $580 \mathrm{~g}$ payload in y-axis between -0.2 to $1.2 \mathrm{~m}$ in the lab. The test results were obtained and plotted in Fig. 6. Specifically, Fig. 6(d) shows the to and fro lateral translational trajectory for the transportation of the payload. In both cases, the drones are able to transport the payload in a similar manner even when they are unevenly distributing the lifting force of the payload. The lifting force was distributed via changing the vertical spatial configuration of the drones relative to each other which is shown in Fig. 6(b). The lift force data obtained by the force sensors is presented in Fig. 6(h). Fig. 6(f) shows the relative trajectories of the drones in both cases. The instantaneous power consumption for both drones is shown in Fig. 6(j), which shows similar values as compared to the simulation results shown in Fig. 6(i) and the resulting energy consumption difference is found to be $6 \%$ which is very close to the predicted value of $6.2 \%$. The error between numerical simulations and experiments, can be attributed to the unaccounted aerodynamic perturbations, caused by the close proximity of drones while in flight. A detailed video of the collaborative transport with load sharing is available at [27].

\section{Conclusion and future work}

This research study proposed an easy to implement multi-UAV collaborative aerial transport strategy with load distribution capability. A load sharing strategy was proposed to deal with uneven battery levels that can ensure the success of the mission. The constraints and limitations of the load sharing strategy were also discussed. A simulation was performed using a hex-rotor-payload model developed using Simulink. The effectiveness of the proposed strategy was demonstrated using an example case. Several experiments were conducted to demonstrate the effect of spatial configuration of the drones on the force distribution and the power consumption. Specifically the experiments demonstrated a $62 \%-38 \%$ force distribution to achieve approximately $6.2 \%$ power consumption distribution using simulations and $6 \%$ power distribution in hardware experiments based on two hex-rotors UAVs. Extended simulations were also performed to achieve a maximum of $81 \%-19 \%$ lift force distribution to distribute the power $33.12 \%$ between the two UAVs. Current work could be expanded to load sharing amongst more than two UAVs. Future work will include the effect of shielding of the thrust airflow of rotors by the payload and how to mitigate such effect. 


\section{Acknowledgements}

This publication is based upon work supported by the Khalifa University of Science and Technology under Award No. RC1-2018-KUCARS.

\section{References}

[1] Lee, H., and Kim, H. J., 2017. "Estimation, control, and planning for autonomous aerial transportation". IEEE Transactions on Industrial Electronics, 64(4), pp. 3369-3379.

[2] Stolaroff, J. K., Samaras, C., ONeill, E. R., Lubers, A., Mitchell, A. S., and Ceperley, D., 2018. "Energy use and life cycle greenhouse gas emissions of drones for commercial package delivery". Nature communications, 9(1), p. 409.

[3] Mohiuddin, A., Tarek, T., Zweiri, Y., and Gan, D., 2020. "A survey of single and multi-uav aerial manipulation". Unmanned Systems.

[4] Kim, S., Seo, H., Choi, S., and Kim, H. J., 2016. "Vision-guided aerial manipulation using a multirotor with a robotic arm”. IEEE/ASME Transactions on Mechatronics, 21(4), pp. 1912-1923.

[5] Mellinger, D., Shomin, M., Michael, N., and Kumar, V., 2013. "Cooperative grasping and transport using multiple quadrotors". In Distributed autonomous robotic systems. Springer, pp. 545-558.

[6] Maza, I., Kondak, K., Bernard, M., and Ollero, A., 2010. "Multi-UAV cooperation and control for load transportation and deployment". Journal of Intelligent and Robotic Systems: Theory and Applications, 57(1-4), pp. 417-449.

[7] Masone, C., Bülthoff, H. H., and Stegagno, P., 2016. "Parameters identification of thrust generation subsystem for small unmanned aerial vehicles". In International Conference on Intelligent Robots and Systems, IEEE, pp. 1623-1630.

[8] Gassner, M., Cieslewski, T., and Scaramuzza, D., 2017. "Dynamic Collaboration without Communication: VisionBased Cable-Suspended Load Transport with Two Quadrotors". In IEEE International Conference on Robotics and Automation (ICRA), IEEE, pp. 5196-5202.

[9] Tagliabue, A., Kamel, M., Verling, S., Siegwart, R., and Nieto, J., 2017. "Collaborative transportation using MAVs via passive force control". In International Conference on Robotics and Automation (ICRA), IEEE, pp. 5766-5773.

[10] Lee, H., Kim, H., and Kim, H. J., 2016. "Planning and control for collision-free cooperative aerial transportation". IEEE Transactions on Automation Science and Engineering.

[11] Loianno, G., and Kumar, V., 2018. "Cooperative transportation using small quadrotors using monocular vision and inertial sensing". IEEE Robotics and Automation Letters, 3(2), pp. 680-687.

[12] Gioioso, G., Franchi, A., Salvietti, G., Scheggi, S., and Prattichizzo, D., 2014. "The flying hand: A formation of UAVs for cooperative aerial tele-manipulation”. In International Conference on Robotics and Automation, IEEE, pp. 43354341.

[13] Mohammadi, M., Franchi, A., Barcelli, D., and Prattichizzo, D., 2016. "Cooperative aerial tele-manipulation with haptic feedback". In International Conference on Intelligent Robots and Systems, IEEE, pp. 5092-5098.

[14] Michael, N., Fink, J., and Kumar, V., 2011. "Cooperative manipulation and transportation with aerial robots". Autonomous Robots, 30(1), pp. 73-86.

[15] Jiang, Q., and Kumar, V., 2013. "The inverse kinematics of cooperative transport with multiple aerial robots". IEEE Transactions on Robotics, 29(1), pp. 136-145.

[16] Fink, J., Michael, N., Kim, S., and Kumar, V., 2011. "Planning and control for cooperative manipulation and transportation with aerial robots". The International Journal of Robotics Research, 30(3), pp. 324-334.

[17] Erskine, J., Chriette, A., and Caro, S., 2019. "Wrench analysis of cable-suspended parallel robots actuated by quadrotor unmanned aerial vehicles". Journal of Mechanisms and Robotics, 11(2), p. 020909.

[18] Jiang, Q., and Kumar, V., 2012. "Determination and stability analysis of equilibrium configurations of objects suspended from multiple aerial robots". Journal of Mechanisms and Robotics, 4(2), p. 021005.

[19] Tognon, M., Gabellieri, C., Pallottino, L., and Franchi, A., 2018. "Aerial co-manipulation with cables: The role of internal force for equilibria, stability, and passivity". IEEE Robotics and Automation Letters, 3(3), pp. 2577-2583.

[20] Estevez, J., and Graña, M., 2017. "Improved Control of DLO Transportation by a Team of Quadrotors". In International Work-Conference on the Interplay Between Natural and Artificial Computation, Springer, pp. 117-126.

[21] Pizetta, I. H. B., Brandão, A. S., and Sarcinelli-Filho, M., 2016. "Cooperative quadrotors carrying a suspended load". In International Conference on Unmanned Aircraft Systems (ICUAS), 2016, IEEE, pp. 1049-1055.

[22] Gimenez, J., Gandolfo, D. C., Salinas, L. R., Rosales, C., and Carelli, R., 2018. "Multi-objective control for cooperative payload transport with rotorcraft UAVs". ISA transactions, 80, pp. 491-502.

[23] Dai, X., Quan, Q., Ren, J., and Cai, K.-Y., 2018. "Efficiency optimization and component selection for propulsion systems of electric multicopters". IEEE Transactions on Industrial Electronics.

[24] Morbidi, F., Cano, R., and Lara, D., 2016. "Minimum-energy path generation for a quadrotor UAV". In IEEE International Conference on Robotics and Automation (ICRA), IEEE, pp. 1492-1498.

[25] Mohiuddin, A., Tarek, T., Zweiri, Y., and Dongming, G., 2019. "UAV payload transportation via RTDP based optimized velocity profiles". Energies, 12(16). 
[26] Filatov, D. M., Friedrich, A. I., and Devyatkin, A. V., 2017. "Parameters identification of thrust generation subsystem for small unmanned aerial vehicles". In II International Conference on Control in Technical Systems (CTS), IEEE, pp. 381-383.

[27] Mohiuddin, A. Youtube video title: Energy Estimation and distribution in Dual-UAV collaborative transportation through load sharing.

[28] Bin Junaid, A., Diaz De Cerio Sanchez, A., Betancor Bosch, J., Vitzilaios, N., and Zweiri, Y., 2018. "Design and implementation of a dual-axis tilting quadcopter". Robotics, 7(4), p. 65.

[29] Pengjie, D., and Yang, S., 2012. "Nonlinear modeling of microminiature multi-rotor aircraft". Ordnance Industry Automation, 6, p. 006. 


\section{List of Figures}

1 Payload with respect to body and world frame along with anchor points $\ldots \ldots \ldots \ldots$

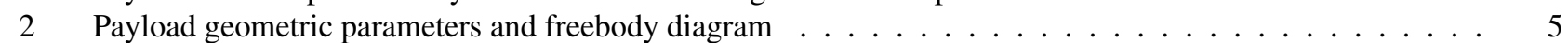

3 Sensitivity of load-distribution potential vs tilt $(\alpha)$, for various aspect ratios (AR) . . . . . . . . . . 6

4 Centralized co-ordinated motion collaboration strategy for multi-UAV payload transportation . . . . . . . 7

5 A complete description of numerical simulation model components used for the verification of load distribu-

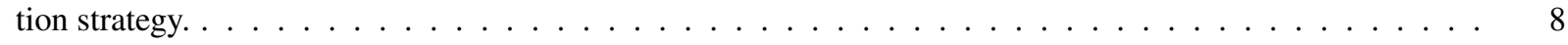

6 First and second column represent similar experimental and simulation scenarios _ . . . . . . . . . 9

7 A description of the hardware used in the experiments. The payload, hex-rotors, gripper with force sensor and EPM is also shown . . . . . . . . . . . . . . . . . . . . . . 11

8 Instantaneous power and energy consumption by both drones, with and without payload, where dashed lines

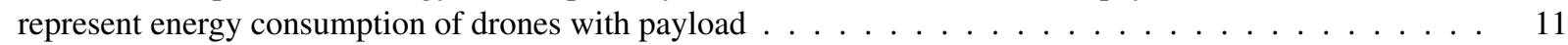

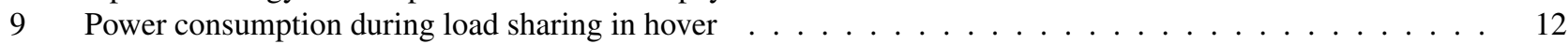

\section{List of Tables}

1 Payload attitude angles for lift load distribution $\ldots \ldots \ldots \ldots \ldots \ldots \ldots \ldots$

2 Extended simulations with various payload shapes and energy states $\ldots \ldots \ldots \ldots$ 